\title{
Robust Speed Tracking of Networked PMSM Servo Systems with Uncertain Feedback Delay and Load Torque Disturbance
}

\author{
Qiang Ling, ${ }^{1}$ Jing Li, ${ }^{1}$ and Haojiang Deng ${ }^{2}$ \\ ${ }^{1}$ Department of Automation, University of Science and Technology of China, Hefei, Anhui 230027, China \\ ${ }^{2}$ National Network New Media Engineering Research Center, Institute of Acoustics, \\ Chinese Academy of Science, Beijing 100190, China \\ Correspondence should be addressed to Qiang Ling, qling@ustc.edu.cn
}

Received 28 February 2012; Revised 30 March 2012; Accepted 31 March 2012

Academic Editor: Baocang Ding

Copyright (C) 2012 Qiang Ling et al. This is an open access article distributed under the Creative Commons Attribution License, which permits unrestricted use, distribution, and reproduction in any medium, provided the original work is properly cited.

\begin{abstract}
This paper considers a class of networked Permanent Magnet Synchronous Motors (PMSMs), whose feedback loops are closed over a shared data network. Although the installation and maintenance cost of the networked PMSM system can be lowered by replacing the conventional point-to-point feedback cables with a network, the network packet dropouts and transmission delay may degrade the system's performance and even destabilize it. The load torque disturbance is another source to deteriorate the PMSM system's performance. To investigate the effects of the data network and the torque disturbance on the speed tracking of a PMSM system is one major task of this paper. In particular, we derive a sufficient stability condition for this system in an LMI (linear matrix inequality) form and provides a way to bound the system's $H_{\infty}$ performance. Moreover, we adopt an iterative LMI method to design the speed controller of the PMSM system, which can robustly guarantee stability and performance against the network-induced delays, packet dropouts, and the torque disturbance. Simulations are done to verify the effectiveness of the obtained results.
\end{abstract}

\section{Introduction}

In the high-performance applications, such as robotics, aeronautic devices, and precision machine tools, the positioning accuracy is required to be higher and higher so that an alternative better than the traditional induction motors is needed. The permanent magnet synchronous motor (PMSM) is one wise choice to meet this accuracy challenge in the lowto-medium power servo systems. Because the PMSM's rotor is a permanent magnet and the flux linkage is constant [1], it possesses many advantages, like superior power density, large torque-to-inertia ratio, and high efficiency. Consequently, the PMSM has received 
widespreading acceptance in industrial applications and is recognized as one of the key components in automation applications.

In PMSM applications, speed tracking is of great importance [2]. The existence of the load torque disturbance, however, makes speed tracking a rather tough task. In fact, it is impossible to measure the disturbance directly in real application. One way is to estimate the disturbance and actively cancel it [3]. Such methods may complicate the system design and increase the system's cost. We take another way, that is, designing a robust controller that can guarantee the system's performance is less sensitive to the disturbance.

Conventionally, a PMSM servo system includes a small number of PMSMs, which are connected to controllers by point-to-point cables to close the control loops. The modern industry, however, demands more and more PMSMs that could be located in geographically separated areas. Thus the direct cable connection architecture is inconvenient for the installation and maintenance. Instead, we can close the feedback loops of PMSMs over a digital network to form a networked control system (NCS), which is a newly developed technology being able to reduce the system's wiring cost, simplify the system's diagnosis and maintenance, and improve the system's agility [4]. The use of network will, however, induce intermittent losses and delays of the feedback information, may deteriorate the system's performance, and even cause instability [5]. There are many results on NCSs, some of which are introduced here (see more in the survey [6]). Reference [7] investigates the problem of robust stabilization and disturbance for the NCSs with random communication networkinduced delays. Reference [8] Considers $H_{\infty}$ output tracking for NCSs with delays and data packet dropouts. In [9], the discrete-time NCSs is studied and a state feedback controller with less conservatism is given. Moreover, a modified optimization algorithm is also proposed in [9] to cope with Bilinear Matrix Inequalities (BMIs).

As an important field of NCSs, networked servo systems are also well studied in recent years. In [10], fuzzy logic control is used to enhance the performance of networked servo systems with delays. To compensate the noise in the transmission channels, two-degrees-offreedom control is utilized in [11]. Predictive control [12] is implemented to suppress the negative effects of delays and dropouts induced by the network. These results mainly focus on servo systems with DC motors or AC induction motors. Nevertheless, PMSM, as one of the main actuators in servo systems, is rarely mentioned in the previous NCS research. Due to its great potential in the industry, the networked PMSM servo systems could be one of the hot research topics in the future. Though a networked PMSM servo system has the same problems of data packet dropouts and transmission delay as typical networked servo systems, it has its own characteristics due to the employment of field-orientation mechanism and the special motor structures [13]. So when we design a controller for such a system, the specific properties should be taken into account to achieve less conservative results. The load torque disturbance, as a common disturbance in applications, should also be considered in the networked PMSM servo system.

This paper is organized as follows. In Section 2, we establish the mathematical model for the networked PMSM servo systems, which suffers the uncertain network-induced delay and data dropouts as well as the load torque disturbance. A sufficient stability condition of the system is given and proven in Section 3 based on the Lyapunov-Krasovskii techniques. Moreover, a bound on the system's $H_{\infty}$ performance is also provided in Section 3. An iterative LMI method is proposed to design a robust controller in Section 4, which can guarantee both the stability and performance under the torque perturbation and the network-induced delay and dropouts. Simulations are done in Section 5 to verify the effectiveness of the obtained results. Some final remarks are placed in Section 6. 


\section{Problem Formulation}

\subsection{PMSM Dynamics}

In the synchronously rotating rotor $d-q$ coordinate, a PMSM drive can be modeled as [2]

$$
\begin{gathered}
u_{d}=R i_{d}+L_{d} \frac{d i_{d}}{d t}-n_{p} \omega L_{q} i_{q}, \\
u_{q}=R i_{q}+L_{q} \frac{d i_{q}}{d t}+n_{p} \omega L_{d} i_{d}+n_{p} \omega \phi_{a} \\
J \frac{d \omega}{d t}+B_{0} \omega+T_{l}=n_{p} \phi_{a}
\end{gathered}
$$

where $\omega$ is the rotating speed; $i_{d}$ and $i_{q}$ are the $d$ - and $q$-axes stator currents, respectively; $u_{d}$ and $u_{q}$ are the $d$ - and $q$-axes stator voltages, respectively; $R$ is the stator resistance per phase; $L_{d}$ and $L_{q}$ are the $d$ - and $q$-axes stator inductances, respectively, and $L_{d}=L_{q}=L$ in the surface-mounted PMSM; $n_{p}$ is the number of poles; $\phi_{a}=\sqrt{3 / 2} \phi_{f}$, with $\phi_{f}$ being the flux linkage of the permanent magnet rotor; $J$ is the total moment of inertia of the motor and load, and $B_{0}$ is the friction coefficient of the motor; $T_{l}$ is the load torque.

A well-known strategy for a PMSM drive is the field-oriented vector control approach. Under this scheme, a practical structure of cascaded control loops, including a speed loop and two current loops, is usually employed [14]. In order to approximately eliminate the coupling between the $d$ - and $q$-axes currents, the $d$-axis reference current $i_{d}^{*}$ is set at zero and $i_{d}$ is regulated via a PI controller, as is shown in Figure 1.

Design the current controller of the $q$-axis as

$$
u_{q}=R i_{q r}+n_{p} \phi_{a} \omega,
$$

where $i_{q r}$ is the reference current of the $q$-axis, which is computed by the speed controller. Then the state-space equation of a PMSM can be represented as

$$
\left[\begin{array}{l}
\frac{d i_{q}}{d t} \\
\frac{d \omega}{d t}
\end{array}\right]=\left[\begin{array}{cc}
-\frac{R}{L} & 0 \\
\frac{n_{p} \phi_{a}}{J} & -\frac{B_{0}}{J}
\end{array}\right]\left[\begin{array}{l}
i_{q} \\
\omega
\end{array}\right]+\left[\begin{array}{c}
\frac{R}{L} \\
0
\end{array}\right] i_{q r}+\left[\begin{array}{c}
0 \\
-\frac{T_{l}}{J}
\end{array}\right] .
$$

To proceed further, we need to make the following two definitions.

Definition 2.1. The tracking errors of speed, the $q$-axis current $i_{q}$, and the reference current $i_{q r}$ are defined as

$$
e(t)=\omega^{*}-\omega, \quad e_{q}(t)=i_{q}^{*}-i_{q}, \quad e_{q r}(t)=i_{q r}^{*}-i_{q r},
$$

where $\omega^{*}, i_{q}^{*}, i_{q r}^{*}$ are the corresponding reference values. 


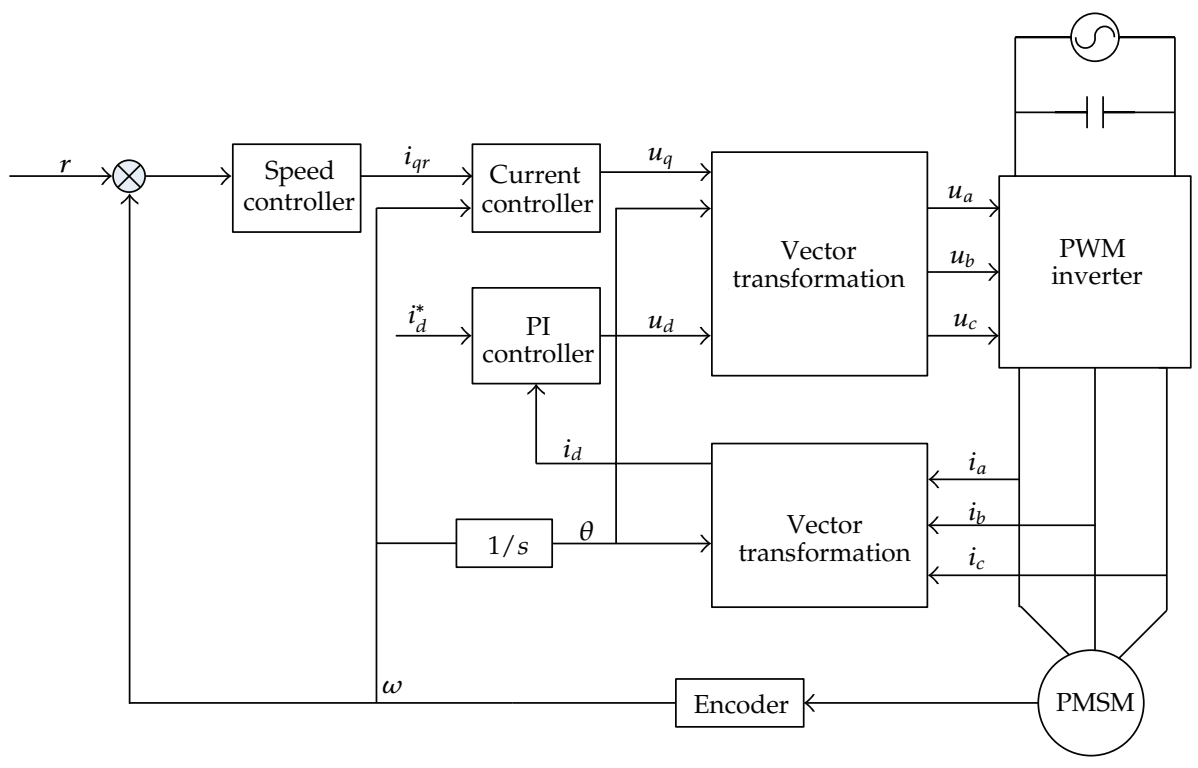

Figure 1: Configuration of a PMSM Servo System.

as

By Definition 2.1, the state-space equation (2.3) can be rewritten into a compact form

$$
\dot{x}(t)=A x(t)+B(u(t)+d(t))
$$

where

$$
\begin{gathered}
x(t)=\left[e_{q}(t) e(t)\right]^{T}, \quad u(t)=\left[\begin{array}{ll}
e_{q r}(t) & 0
\end{array}\right]^{T}, \quad d(t)=\left[\begin{array}{ll}
0 & \Delta T_{l}(t)
\end{array}\right]^{T}, \\
A=\left[\begin{array}{cc}
-\frac{R}{L} & 0 \\
\frac{n_{p} \phi_{a}}{J} & -\frac{B_{0}}{J}
\end{array}\right], \quad B=\left[\begin{array}{ll}
\frac{R}{L} & 0 \\
0 & \frac{1}{J}
\end{array}\right] .
\end{gathered}
$$

$\Delta T_{l}(t)$ represents the load torque disturbance, which is bounded and perhaps time-varying.

Definition 2.2. The disturbance suppressing performance of the system is evaluated by the following signal:

$$
z(t)=e(t)
$$

We want to design a robust controller for a networked PMSM to satisfy the following requirements.

(1) When the load torque disturbance $d(t)=0$, the system is asymptotically stable under any initial state, namely,

$$
\lim _{t \rightarrow \infty} z(t)=0
$$




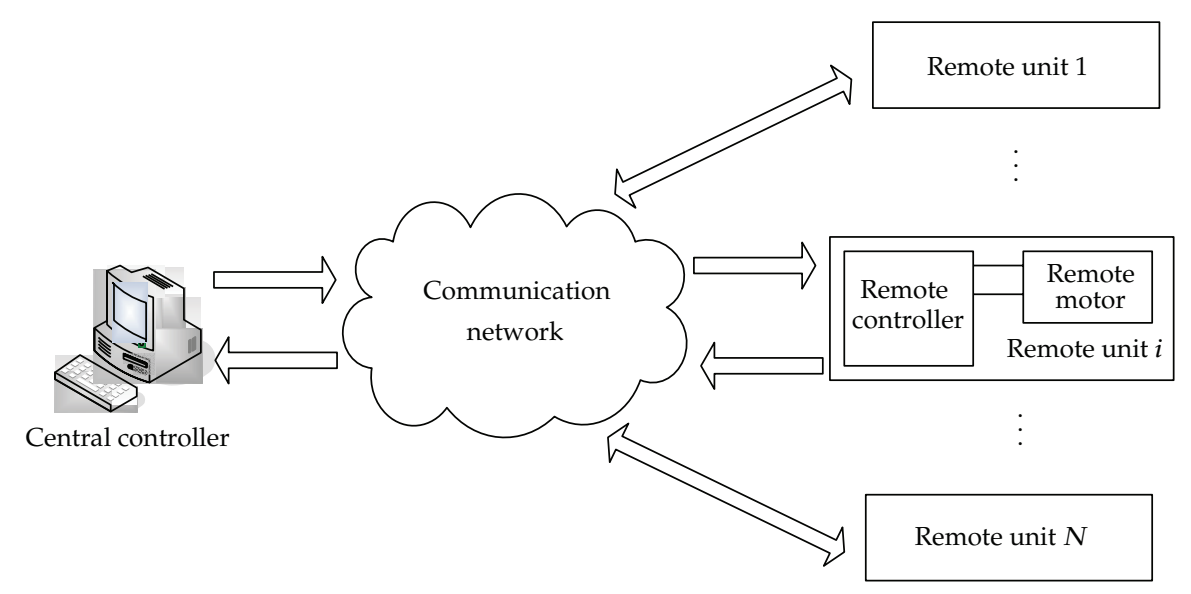

Figure 2: The diagram of a typical networked servo system.

(2) When the load torque disturbance $d(t) \neq 0$, the closed-loop system has the ability to suppress disturbance, namely,

$$
\|z(t)\|_{2} \leq r\|d(t)\|_{2}
$$

where $\|\cdot\|_{2}$ stands for the $L_{2}$ norm of a continuous-time signal and $\gamma$ is a quantitative measure of the disturbance attenuation. The smaller $\gamma$, the better disturbance attenuation.

\subsection{Structure of Networked PMSM Servo Systems}

A typical networked servo system is shown in Figure 2, which can be divided into three parts:

(1) the remote unit containing a remote controller and a remote motor,

(2) the central controller,

(3) the communication network.

The remote unit and the central controller exchange feedback information through the communication network.

When the remote motors are PMSMs, we get a networked PMSM servo system as shown in Figure 3. Each distributed remote controller receives control signals from the communication network and then convert them into PWM signals to drive the motor. It also sends local measurements, such as rotating speed, motor current, and local environment information, back to the central controller via the shared data network. The central controller is usually a sophisticated controller and can provide advanced real-time control strategies to the remote units.

\subsection{System Modeling}

Because of the limited bandwidth of the network, data packet dropouts are unavoidable. When a dropout occurs, it might be more advantageous to drop the old packet and transmit a new one than to retransmit the old one [15]. Network-induced delays are also considered here. The model of the concerned networked PMSM servo system is shown in Figure 4. 


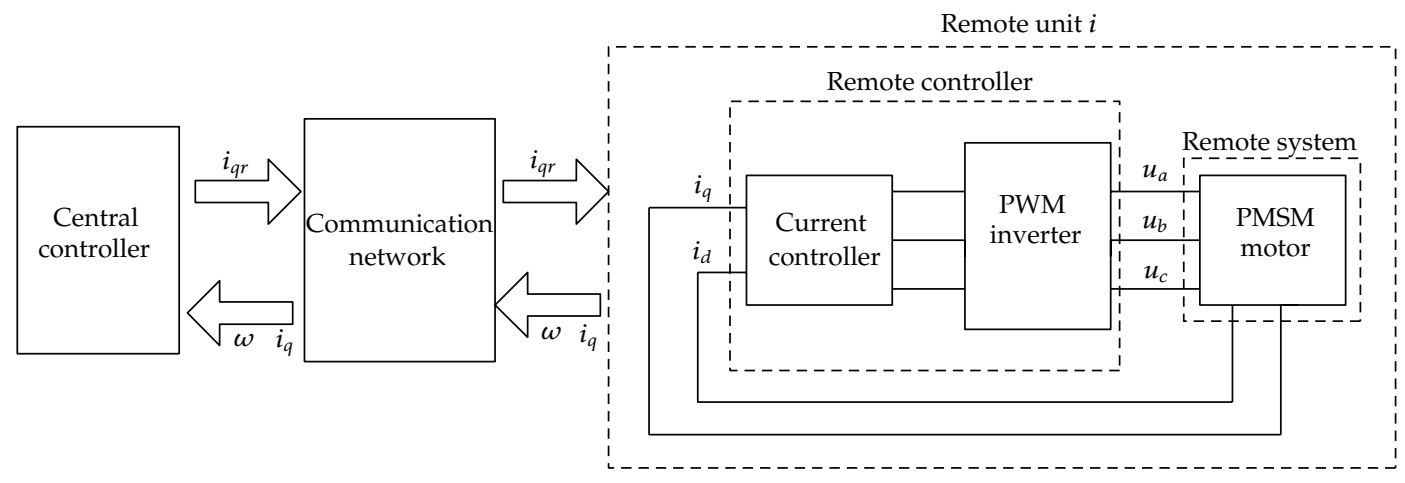

Figure 3: The diagram of a networked PMSM servo system.

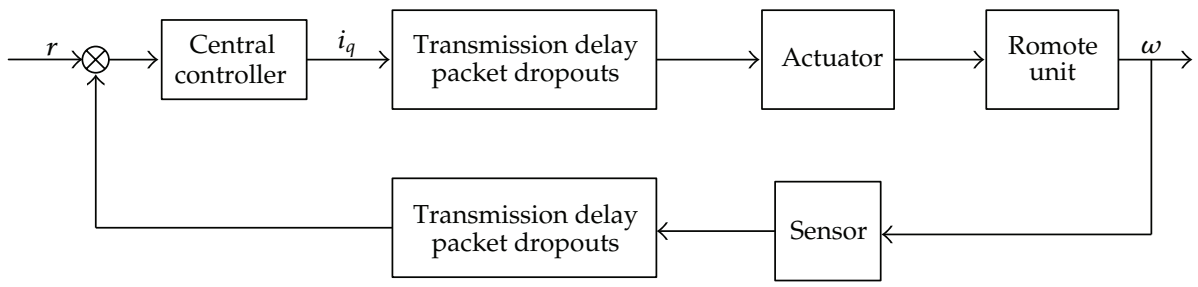

Figure 4: The model of the networked PMSM servo system.

The following assumptions are placed on the networked PMSM servo system.

(1) The sensor is clock driven, and the controller and actuator are event driven.

(2) The sampling period is a positive constant scalar $h$.

(3) The controller-to-actuator and the sensor-to-controller delays are denoted as $\tau_{c a}$ and $\tau_{s c}$, respectively. The state feedback controller is static (see (2.14)). So these two delays can be lumped as

$$
\tau_{k}=\tau_{s c}+\tau_{c a}
$$

Moreover, $\tau_{k}$ is less than $h$, that is, $0 \leq \tau_{k} \leq \bar{\tau} \leq h$, where $\bar{\tau}$ is the upper bound of delays. Due to the static feedback controller, we can assume that the transmission from the controller to the actuator is delay free and all delays come from the transmissions from the sensor to the controller, that is, $\tau_{s c}=\tau_{k}$ and $\tau_{c a}=0$, in Figure 4.

(4) The maximum numbers of the consecutive controller-to-actuator and sensor-tocontroller data dropouts are denoted as $d_{c a}$ and $d_{s c}$, respectively. They can also be lumped as

$$
d_{k}=d_{c a}+d_{s c}
$$

$d_{k}$ is bounded as $0 \leq d_{k} \leq \bar{d}$. Similar to $\tau_{k}, d_{k}$ is also assumed to only come from the transmissions from the sensor to the controller, that is, $d_{s c}=d_{k}$ and $d_{c a}=0$. 
If only delays exist in the system, the sampled signal at $k h(\forall k \in \mathcal{N})$ will arrive at the controller at the time $k h+\tau_{k}$. So the delay is $\eta(t)=t-k h$ and its range is

$$
\eta(t) \in[0, h+\bar{\tau}]
$$

When the data dropouts also exist, they can be treated as delays and yield the following overall delay range:

$$
\eta(t) \in[0,(\bar{d}+1) h+\bar{\tau}]=[0, \bar{\eta}]
$$

It is well known that time-varying delay is more difficult to handle than constant delay from the control system's perspective. The actuator can know the total delay $\eta(t)$ by the time stamping technique. In the present paper, the actuator is assumed to purposefully postpone to implement the received control variable by the time of $\bar{\eta}-\eta(t)$ and yields a constant overall delay of $\bar{\eta}$, which is easier to deal with. We choose a static state feedback controller. Due to the constant delay strategy, our controller takes the following form:

$$
u(t)=K x(t-\bar{\eta}),
$$

where $K$ is the feedback gain to be designed. By substituting (2.14) into (2.5), we get the following state-space equation of the networked PMSM servo system:

$$
\begin{gathered}
\dot{x}(t)=A x(t)+B K x(t-\bar{\eta})+B d, \\
z(t)=C x(t) .
\end{gathered}
$$

\section{Analysis of Networked PMSM Servo Systems}

\subsection{A Sufficient Stability Condition}

In industry applications, the stability of a servo system is crucial. So we first have to guarantee that the system is stable. Here stability means the asymptotic stability when the disturbance is zero, that is, $d(t)=0$. Under the delay and dropout conditions in (2.13), we get the following stability condition, which is expressed in an LMI (linear matrix inequality) form and easy to verify.

Theorem 3.1. Under the given controller gain $K$ and the upper bound $\bar{\eta}>0$ (in (2.13)), the system (2.15) is asymptotically stable if there exist matrices $P>0, Q>0, Z>0, Y$ and $W$ such that the following matrix inequality (3.1) holds:

$$
\left[\begin{array}{cccc}
W_{1} & W_{2} & -\bar{\eta} Y & A^{T} \\
* & W_{3} & -\bar{\eta} W & K^{T} B^{T} \\
* & * & -\bar{\eta} Z & 0 \\
* & * & * & -\bar{\eta}^{-1} Z^{-1}
\end{array}\right]<0
$$

where $W_{1}=P A+A^{T} P+Y+Y^{T}+Q, W_{2}=P B K-Y+W^{T}$, and $W_{3}=-Q-W-W^{T}$. 
Proof. We construct the following Lyapunov-Krasovskii function:

$$
V(t)=V_{1}(t)+V_{2}(t)+V_{3}(t),
$$

where $V_{1}(t)=x^{T}(t) P x(t), V_{2}(t)=\int_{t-\bar{\eta}}^{t} x^{T}(\alpha) Q x(\alpha) d \alpha$, and $V_{3}(t)=\int_{-\bar{\eta}}^{0} \int_{t+\beta}^{t} \dot{x}^{T}(s) Z \dot{x}(s) d s d \beta$. By the Newton-Leibniz formula, we get

$$
x(t-\bar{\eta})=x(t)-\int_{t-\bar{\eta}}^{t} \dot{x}(\alpha) d \alpha .
$$

The derivatives of $V_{1}, V_{2}$, and $V_{3}$ are computed as follows:

$$
\begin{aligned}
& \dot{V}_{1}(t)= 2 x^{T}(t) P[A x(t)+B K x(t-\bar{\eta})] \\
&= 2 x^{T}(t) P(A+B K) x(t)-2 x^{T}(t) P B K \int_{t-\bar{\eta}}^{t} \dot{x}(\alpha) d \alpha \\
&= 2 x^{T}(t) P(A+B K) x(t) \\
&+2 x^{T}(t)(Y-P B K) \int_{t-\bar{\eta}}^{t} \dot{x}(\alpha) d \alpha+2 x^{T}(t-\bar{\eta}) W \int_{t-\bar{\eta}}^{t} \dot{x}(\alpha) d \alpha \\
&-\left[2 x^{T}(t) Y \int_{t-\bar{\eta}}^{t} \dot{x}(\alpha) d \alpha+2 x^{T}(t-\bar{\eta}) W \int_{t-\bar{\eta}}^{t} \dot{x}(\alpha) d \alpha\right] \\
&= 2 x^{T}(t) P(A+B K) x(t)+2 x^{T}(t)(Y-P B K)[x(t)-x(t-\bar{\eta})] \\
&+2 x^{T}(t-\bar{\eta}) W[x(t)-x(t-\bar{\eta})] \\
&-\left[2 x^{T}(t) Y \int_{t-\bar{\eta}}^{t} \dot{x}(\alpha) d \alpha+2 x^{T}(t-\bar{\eta}) W \int_{t-\bar{\eta}}^{t} \dot{x}(\alpha) d \alpha\right] \\
&= \frac{1}{\bar{\eta}} \int_{t-\bar{\eta}}^{t}\left[2 x^{T}(t)(P A+Y) x(t)+2 x^{T}(t)\left(P B K-Y+W^{T}\right) x(t-\bar{\eta})\right. \\
& \dot{V}_{2}(t)= x^{T}(t) Q x(t)-x^{T}(t-\bar{\eta}) Q x(t-\bar{\eta}) \\
&= \frac{1}{\bar{\eta}} \int_{t-\bar{\eta}}^{t}\left[x^{T}(t) Q x(t)-x^{T}(t-\bar{\eta}) Q x(t-\bar{\eta})\right] d \alpha, \\
& \dot{V}_{3}(t)= \int_{-\bar{\eta}}^{0}\left[\dot{x}^{T}(t) Z \dot{x}(t)-\dot{x}(t+\beta) Z \dot{x}(t+\beta)\right] d \beta \\
&= \int_{t-\bar{\eta}}^{t}\left[\dot{x}^{T}(t) Z \dot{x}(t)-\dot{x}(\alpha) Z \dot{x}(\alpha)\right] d \alpha \\
&= \frac{1}{\bar{\eta}} \int_{t-\bar{\eta}}^{t}\left[x^{T}(t) \bar{\eta} A^{T} Z A x(t)+2 x^{T}(t) \bar{\eta} A^{T} Z B K x(t-\bar{\eta})\right. \\
&\left.\quad+x^{T}(t-\bar{\eta}) \bar{\eta} K^{T} B^{T} Z B K x(t-\bar{\eta})-\dot{x}^{T}(\alpha) \bar{\eta} Z \dot{x}(\alpha)\right] d \alpha . \\
& \dot{\eta}(\alpha) W x(t-\bar{\eta})-2 x^{T}(t) \bar{\eta} Y \dot{x}(\alpha)-2 x^{T}(t-\bar{\eta}) \bar{\eta} W \dot{x}(\alpha) \\
&
\end{aligned}
$$


Journal of Applied Mathematics

Finally, we have

$$
\dot{V}(t)=\frac{1}{\bar{\eta}} \int_{t-\bar{\eta}}^{t} \zeta^{T}(t, \alpha) \Phi \zeta(t, \alpha) d \alpha,
$$

where

$$
\zeta(t, \alpha)=\left[\begin{array}{c}
x(t) \\
x(t-\bar{\eta}) \\
\dot{x}(\alpha)
\end{array}\right], \quad \Phi=\left[\begin{array}{ccc}
W_{1}+\bar{\eta} A^{T} Z A & W_{2}+\bar{\eta} A^{T} Z B K & -\bar{\eta} Y \\
* & W_{3}+\bar{\eta} K^{T} B^{T} Z B K & -\bar{\eta} W \\
* & * & -\bar{\eta} Z
\end{array}\right] .
$$

By the Schur complement theorem, we get from (3.1) that there must exist $\epsilon>0$ such that

$$
\Phi<-\epsilon I,
$$

where $I$ represents an identity matrix of an appropriate dimension. Substituting (3.7) into (3.5) yields

$$
\dot{V}(t)<-\epsilon\|x(t)\|^{2}
$$

According to Lyapunov-Krasovskii theorem, if there exist $\epsilon>0$ such that $\dot{V}(t)<-\varepsilon\|x\|^{2}$, the system (2.15) is asymptotic stable. So if the matrix inequality (3.1) holds, the system (2.15) is asymptotically stable. This completes the proof.

\subsection{Robust Performance Analysis}

Definition 3.2. A stable system in (2.15) is said to satisfy the $H_{\infty}$ performance index $\gamma>0$ if under the zero initial condition,

$$
\|z(t)\|_{2} \leq \gamma\|d(t)\|_{2} .
$$

We can verify whether the performance requirement in (3.9) is satisfied through the following theorem.

Theorem 3.3. Under the given controller gain $K$ and the upper bound $\bar{\eta}>0$ (in (2.13)), the system (2.15) satisfies the performance index $\gamma$ in (3.9) if there exist matrices $P>0, Q>0, Z>0, Y$ and $W$ such that the following matrix inequality holds:

$$
\left[\begin{array}{cccccc}
W_{1} & W_{2} & -\bar{\eta} Y & P B & A^{T} & C^{T} \\
* & W_{3} & -\bar{\eta} W & 0 & K^{T} B^{T} & 0 \\
* & * & -\bar{\eta} Z & 0 & 0 & 0 \\
* & * & * & -\gamma^{2} I & B^{T} & 0 \\
* & * & * & * & -\bar{\eta}^{-1} Z^{-1} & 0 \\
* & * & * & * & * & -I
\end{array}\right]<0,
$$

where $W_{1}=P A+A^{T} P+Y+Y^{T}+Q, W_{2}=P B K-Y+W^{T}$, and $W_{3}=-Q-W-W^{T}$. 
Proof. Equation (3.10) implies (3.1). By Theorem 3.1, we know the system is stable. Define $J_{z d}=\int_{0}^{\infty}\left[z^{T}(t) z(t)-\gamma^{2} d^{T}(t) d(t)\right] d t$. $J_{z d}$ can be modified into

$$
J_{z d}=\int_{0}^{\infty}\left[z^{T}(t) z(t)-r^{2} d^{T}(t) d(t)+\dot{V}(t)\right] d t+\left.V(t)\right|_{t=0}-\left.V(t)\right|_{t=\infty} .
$$

Under the zero initial condition, $\left.V(t)\right|_{t=0}=0$. Because of $\left.V(t)\right|_{t=\infty} \geq 0$, we get

$$
\begin{aligned}
J_{z d} & \leq \int_{0}^{\infty}\left[z^{T}(t) z(t)-r^{2} d^{T}(t) d(t)+\dot{V}(t)\right] d t \\
& \leq \frac{1}{\bar{\eta}} \int_{0}^{\infty} \int_{t-\bar{\eta}}^{t}\left[\begin{array}{c}
x(t) \\
x(t-\bar{\eta}) \\
\dot{x}(\alpha) \\
d(t)
\end{array}\right]^{T} \Xi\left[\begin{array}{c}
x(t) \\
x(t-\bar{\eta}) \\
\dot{x}(\alpha) \\
d(t)
\end{array}\right],
\end{aligned}
$$

where

$$
\Xi=\left[\begin{array}{cccc}
W_{1}+C^{T} C & W_{2} & -\bar{\eta} Y & P B \\
* & W_{3} & -\bar{\eta} W & 0 \\
* & * & -\bar{\eta} Z & 0 \\
* & * & * & -\gamma^{2} I
\end{array}\right]+\left[\begin{array}{c}
A^{T} \\
K^{T} B^{T} \\
0 \\
B^{T}
\end{array}\right] \bar{\eta} Z\left[\begin{array}{c}
A^{T} \\
K^{T} B^{T} \\
0 \\
B^{T}
\end{array}\right]^{T}
$$

By the Schur complement theorem, we know that (3.10) implies $\Xi<0$. Therefore, $J_{z \omega}<0$, that is,

$$
\int_{0}^{\infty}\left[z^{T}(t) z(t)-r^{2} d^{T}(t) d(t)\right] d t<0
$$

After simple manipulations, the above equation yields

$$
\|z(t)\|_{2}^{2} \leq r^{2}\|d(t)\|_{2}^{2}
$$

Equation (3.15) is equivalent to (3.9).

In (3.9), the left side variable $z(t)$ and the right one $d(t)$ have different units. So the ratio between them, $\gamma$, does not have a clear physical meaning. In order to overcome this difficulty, we introduce the following relative sensitive functions.

Definition 3.4. The sensitive functions of the speed tracking error and the load torque disturbance are defined as

$$
S_{z}=\frac{\|Z\|_{2}}{\omega_{\text {ref }}}, \quad S_{T}=\frac{\left\|\Delta T_{l}\right\|_{2}}{T_{l 0}}
$$

where $\omega_{\text {ref }}$ is the reference tracking speed and $T_{l 0}$ is the nominal load torque. 
Based on the above definition, (3.15) can be rewritten into

$$
S_{z} \leq \tilde{\gamma} S_{T}
$$

where $\tilde{\gamma}=\left(T_{l 0} / \omega_{\text {ref }}\right) \gamma$.

Remark 3.5. In (3.17), both units of $S_{z}$ and $S_{T}$ are percentage. So (3.17) means how much percent of load torque disturbance yields how much percent of speed tracking error. $\tilde{\gamma}$ is exactly the gain between two percentage variables $\left(S_{z}\right.$ and $\left.S_{T}\right)$ and can quantitatively reflect the capability to attenuate the load torque disturbance attenuation. $\tilde{\gamma}$ is determined by the system's delay in (2.13) and the controller gain $K$ in (2.14). Although we cannot change the system's delay, we do have freedom to choose an appropriate $K$ to yield a better (smaller) $\tilde{\gamma}$, which is the major task of the next section.

\section{The Design of the Robust Controller}

When we design the robust controller, the controller gain $K$ is unknown. So the matrix inequalities in Theorems 3.1 and 3.3 are bilinear matrix inequalities (BMIs). As a result, we cannot find a maximum $\bar{\eta}$ or the minimum $\gamma$ using convex optimization algorithms. In the subsequent part, we propose some methods to resolve this issue.

Define $X=P^{-1}$ and $\Delta=\operatorname{diag}\{X, X, X, I, I, I\}$. Pre- and postmultiply (3.10) by $\Delta$, we obtain

$$
\left[\begin{array}{cccccc}
\widetilde{W}_{1} & \widetilde{W}_{2} & -\bar{\eta} \tilde{Y} & B & X A^{T} & X C^{T} \\
* & \widetilde{W}_{3} & -\bar{\eta} \widetilde{W} & 0 & F^{T} B^{T} & 0 \\
* & * & -\bar{\eta} X S^{-1} X & 0 & 0 & 0 \\
* & * & * & -\gamma^{2} I & B^{T} & 0 \\
* & * & * & * & -\bar{\eta}^{-1} S & 0 \\
* & * & * & * & * & -I
\end{array}\right]<0,
$$

where $\widetilde{W}_{1}=A X+X A^{T}+\tilde{Y}+\tilde{Y}^{T}+\widetilde{Q}, \widetilde{W}_{2}=B F-\tilde{Y}+\widetilde{W}^{T}, \widetilde{W}_{3}=-\widetilde{Q}-\widetilde{W}-\widetilde{W}^{T}, \tilde{Y}=X Y X$, $\widetilde{Q}=X Q X, \widetilde{W}=X W X, F=K X$, and $S=Z^{-1}$.

Define a matrix variable $M<X S^{-1} X$, then matrix inequality (4.1) is equivalent to the combination of matrix inequalities (4.2), (4.3) and(4.4)

$$
\left[\begin{array}{cccccc}
\widetilde{W}_{1} & \widetilde{W}_{2} & -\bar{\eta} \tilde{Y} & B & X A^{T} & X C^{T} \\
* & \widetilde{W}_{3} & -\bar{\eta} \widetilde{W} & 0 & F^{T} B^{T} & 0 \\
* & * & -\bar{\eta} M & 0 & 0 & 0 \\
* & * & * & -\gamma^{2} I & B^{T} & 0 \\
* & * & * & * & -\bar{\eta}^{-1} S & 0 \\
* & * & * & * & * & -I
\end{array}\right]<0,
$$




$$
\begin{gathered}
{\left[\begin{array}{cc}
-Z & P \\
P & -N
\end{array}\right]<0,} \\
S=Z^{-1}, \quad N=M^{-1}, \quad P=X^{-1} .
\end{gathered}
$$

Based on the above transformation, a robust controller can be designed as follows.

Theorem 4.1. Under the given $\bar{\eta}>0$ and $\gamma>0$, the system (2.15) satisfies the performance index $\gamma$ if there exist matrices $X>0, P>0, Q>0, Z>0, M>0, S>0, N>0, Y$ and $W$ such that matrix inequalities (4.2), (4.3) and (4.4) hold. In this case, a robust $H_{\infty}$ state feedback controller gain can be chosen as $K=F X^{-1}$.

It is noted that the conditions in Theorem 4.1 are not LMI because of the inverse matrix constraints in (4.3) and (4.4). Fortunately, there are some methods to efficiently solve these inequalities. In [16], a method is given to obtain the suboptimal delay $\bar{\eta}$ or the suboptimal $\gamma$ by setting $S=X$. With more computational efforts, better results can be obtained by an iterative algorithm in [17]. That iterative algorithm is called cone complementary linearization (CCL) method. By adopting the CCL method, we get the following algorithm to cope with the nonlinear minimization problem subject to LMIs.

Algorithm 4.2. There are 4 steps.

Step 1. Choose a sufficiently large initial variable $\gamma>0$ such that there exists a feasible solution to matrix inequalities (4.2) and (4.3) and (4.4). Set $\gamma_{\min }=\gamma$.

Step 2. Find a feasible set $\left(X_{0}, P_{0}, Q_{0}, Z_{0}, M_{0}, S_{0}, N_{0}, Y_{0}, W_{0}\right)$ satisfying (4.2) and (4.3), and the matrix inequality in (4.5) (One way to get a feasible set is by setting $S=X$ as the aforementioned suboptimal solution). Set $k=0$,

$$
\left[\begin{array}{cc}
S & I \\
I & Z
\end{array}\right]<0, \quad\left[\begin{array}{cc}
N & I \\
I & M
\end{array}\right]<0, \quad\left[\begin{array}{cc}
P & I \\
I & X
\end{array}\right]<0 .
$$

Step 3. Solve the LMI problem in (4.6),

$$
\operatorname{Min}_{X, P, Q, Z, M, S, N, Y, W} \operatorname{tr}\left(S_{k} Z+S Z_{k}+N M_{k}+P_{k} X+P X_{k}\right)
$$

s.t. equations (4.2),(4.3), and (4.5).

Set $X_{k+1}=X, P_{k+1}=P, Q_{k+1}=Q, Z_{k+1}=Z, M_{k+1}=M, S_{k+1}=S, N_{k+1}=N, Y_{k+1}=Y$, and $W_{k+1}=W$.

Step 4. If (3.10) holds, then set $\gamma_{\text {min }}=\gamma$ and return to Step 2 after decreasing $\gamma$ to some extent. If (3.10) is not satisfied within a specified number of iterations, to say $k_{\max }$, then we stop the above iterations. Otherwise, set $k=k+1$ and go back to Step 3 . 
Table 1: The nominal parameters of a PMSM.

\begin{tabular}{lccc}
\hline$R$ & $L$ & $\phi_{f}$ & $B_{0}$ \\
\hline $2.875 \Omega$ & $0.0085 \mathrm{H}$ & $0.0816 \mathrm{~Wb}$ & $0.00185 \mathrm{~N} \cdot \mathrm{m} \cdot \mathrm{s}$ \\
\hline$J$ & $n_{p}$ & $T_{l}$ & \\
\hline $0.0008 \mathrm{~N} \cdot \mathrm{m} \cdot \mathrm{s}$ & 4 & $2 \mathrm{~N} \cdot \mathrm{m}$ & \\
\hline
\end{tabular}

Table 2: The relationship between the speed tracking error and $\bar{\eta}$.

\begin{tabular}{lcccccc}
\hline $\begin{array}{l}\text { Speed error } \\
\text { percentage }\end{array}$ & $6 \%$ & $5.6 \%$ & $5.2 \%$ & $4.8 \%$ & $4.4 \%$ & $4.0 \%$ \\
\hline $\bar{\eta}$ & 0.082 & 0.075 & 0.068 & 0.061 & 0.055 & 0.049 \\
\hline $\begin{array}{l}\text { Speed error } \\
\text { percentage }\end{array}$ & $3.6 \%$ & $3.2 \%$ & $2.8 \%$ & $2.4 \%$ & $2.0 \%$ & \\
\hline $\bar{\eta}$ & 0.043 & 0.037 & 0.032 & 0.026 & 0.005 & \\
\hline
\end{tabular}

\section{Simulation Results}

To verify the results in Theorems 3.1, 3.3, and 4.1, a MATLAB/SIMULINK simulation platform of networked PMSM servo systems is built up, which is shown in Figure 1. The sampling period $h$ is $10 \mathrm{~ms}$. The tracking reference speed is set to $1500 \mathrm{r} / \mathrm{min}$. The nominal parameters of a PMSM is shown in Table 1.

In the following simulations, the load torque disturbance is no larger than $30 \%$ of the nominal value. According to Theorem 4.1 and Definition 3.4, we obtain the relationship between the speed tracking error and $\bar{\eta}$, which is demonstrated in Table 2.

Remark 5.1. The data in Table 2 shows the relationship between the speed tracking error and the maximum delay $\bar{\eta}$. When the networked PMSM servo system needs higher tracking accuracy, it can tolerate less transmission delay and data packet dropouts. If the speed tacking error is less than $2.0 \%, \bar{\eta}$ is equal to $0.005 \mathrm{~s}$, which means that the system cannot tolerate even one data packet dropout under this circumstance.

In the sequel, we simulate 3 cases to demonstrate the effectiveness of the obtained results. In these simulations, the maximum relative speed tracking error is $5 \%$, and the load torque disturbance is no larger than $30 \%$ of the nominal value. According to Definition 3.4, we get

$$
r \leq \gamma_{\min }=125
$$

By Theorem 4.1 (with $\gamma=\gamma_{\min }$ ), we try to maximize $\bar{\eta}$ and reach a suboptimal solution with $\bar{\eta}=0.064 \mathrm{~s}$ and the corresponding controller gain of $K=[0,-0.0212]$.

\subsection{Stability of System}

Case 1. The first simulation is to verify the stability of the networked PMSM servo system. $\bar{\eta}$ is set to $0.064 \mathrm{~s}$. Figure 5 shows the speed tracking error trajectory. After approximate $0.15 \mathrm{~s}$, the speed tracking error is almost zero. So this result demonstrates the correctness of the stability condition in Theorem 3.1. 


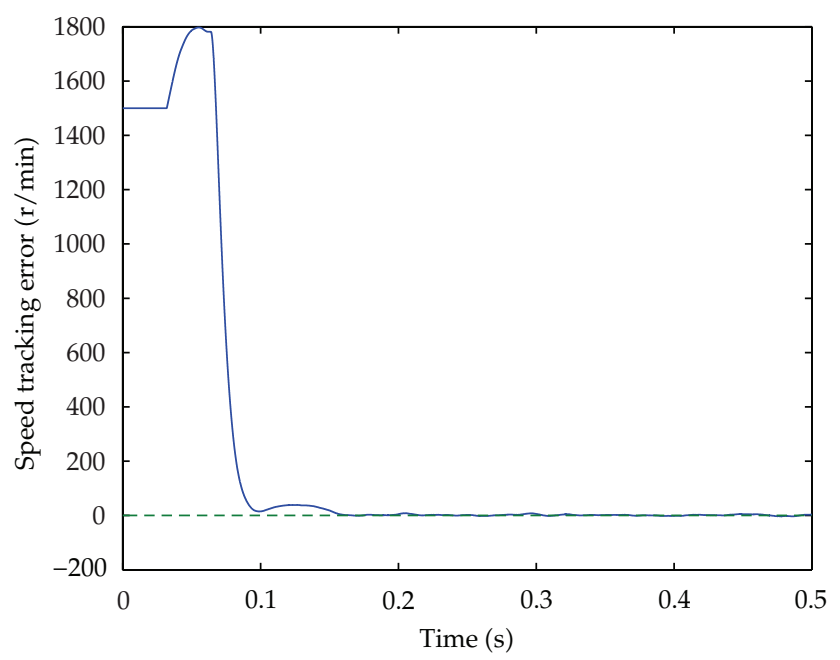

Figure 5: The speed tracking error.

\subsection{Robustness against Transmission Delay and Data Dropouts}

Case 2. In the second case, performance comparison is made between two approaches, the robust controller proposed in this paper and the PID controller.

\section{The PID Controller}

Its parameters are tuned to perform well under the network-delay-free situation. In the simulation, the network-induced delay is set to $0.1 \mathrm{~h}(=0.001 \mathrm{~s})$.

\section{Robust Controller}

The robust controller is then applied as the central controller to regulate the speed loop. The network-induced delay $\bar{\eta}$ is set to be $0.064 \mathrm{~s} . \bar{\eta}=0.064 \mathrm{~s}=6.4 \mathrm{~h}$ means that the system can tolerate 5 dropouts among any 6 consecutive data packets.

The results of this case are shown in Figure 6.

Although the PID controller suffers a much smaller delay than the robust controller, it generates much worse tracking performance than the robust controller. The reason lies in that the design of the robust controller takes the delay into account, while the design of the PID controller does not. So the effectiveness of Theorems 3.3 and 4.1 is confirmed.

\subsection{Robustness against Load Torque Disturbance}

Case 3. The purpose of the third simulation is to verify that the proposed robust controller can effectively suppress the load torque disturbance. Under the worst condition of five consecutive data packet dropouts and network-induced delay up to $0.4 \mathrm{~h}$, a $30 \%$ load torque disturbance occurs at time $0.5 \mathrm{~s}$ and disappears at time $0.55 \mathrm{~s}$. Figure 7 shows the simulation results. 


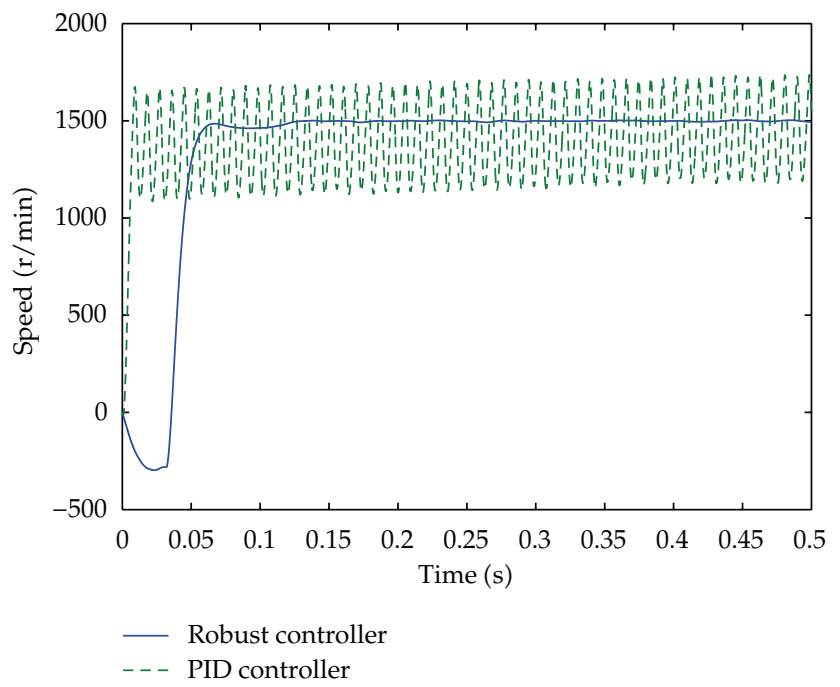

Figure 6: Speed response under the network delays and dropouts.

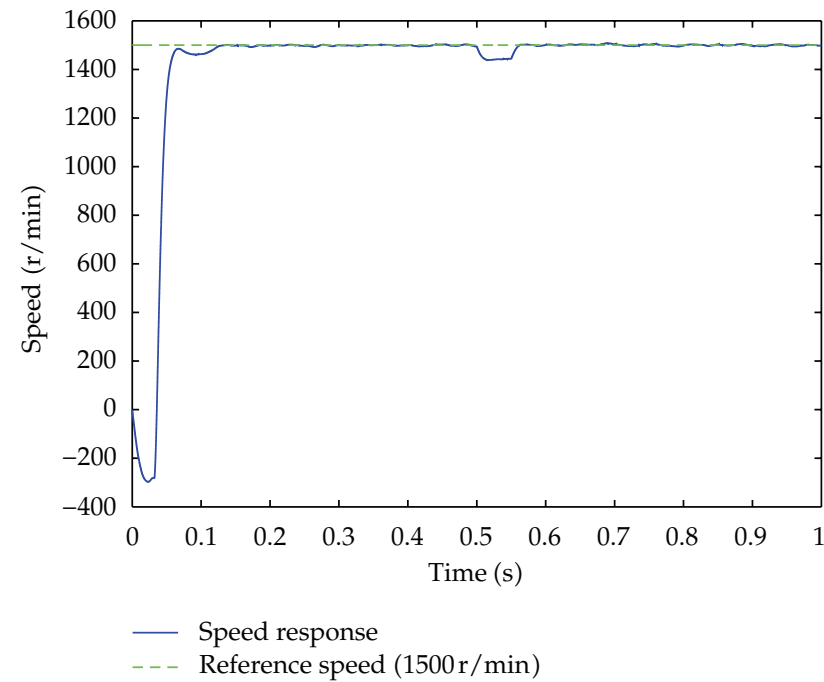

Figure 7: Speed response under the network delays and dropouts and the load torque disturbance.

As we see in Figure 7, when the load torque disturbance starts at time $0.5 \mathrm{~s}$, the speed deviates from the reference speed of $1500 \mathrm{r} / \mathrm{min}$. However, after the disturbance disappears at time $0.55 \mathrm{~s}$, the speed of PMSM quickly returns back to the reference speed in about $0.03 \mathrm{~s}$. From the speed response curve, we can also see that the speed tracking error is less than $5 \%$ during the whole process. These simulation results confirm that the robust controller designed in this paper satisfies the accuracy demand and can effectively suppress the load torque disturbance.

\section{Conclusion}

The networked PMSM servo system has a promising future in industry applications. However, its performance may be degraded by the network delays and data packet dropouts. 
The load disturbance is also a detrimental factor for the control performance. In this paper, we propose a sufficient stability condition by the Lyapunov-Krasovskii method, quantitatively investigate the robustness of the system's performance against the load torque disturbance, and give a way to design a robust controller, which can either tolerate larger network delay or give better $H_{\infty}$ performance. The simulations are done to verify the correctness of the stability result and demonstrate the superiority of the obtained controller in terms of performance robustness against the data packet dropouts and transmission delay as well as the load torque disturbance.

\section{Acknowledgments}

This paper was supported in part by the National Natural Science Foundation of China (60904012), the Program for New Century Excellent Talents in University (NCET0-10-0917), and the Doctoral Fund of Ministry of Education of China (20093402120017).

\section{References}

[1] C. F. J. Kuo, C. H. Hsu, and C. C. Tsai, "Control of a permanent magnet synchronous motor with a fuzzy sliding-mode controller," International Journal of Advanced Manufacturing Technology, vol. 32, no. 7-8, pp. 757-763, 2007.

[2] S. S. Yang and Y. S. Zhong, "Robust speed tracking of permanent magnet synchronous motor servo systems by equivalent disturbance attenuation," IET Control Theory and Applications, vol. 1, no. 3, pp. 595-603, 2007.

[3] K. H. Kim, I. C. Baik, G. W. Moon, and M. J. Youn, "A current control for a permanent magnet synchronous motor with a simple disturbance estimation scheme," IEEE Transactions on Control Systems Technology, vol. 7, no. 5, pp. 630-633, 1999.

[4] W. Zhang, M. S. Branicky, and S. M. Phillips, "Stability of networked control systems," IEEE Control Systems Magazine, vol. 21, no. 1, pp. 84-99, 2001.

[5] P. Seiler and R. Sengupta, "An $H_{\infty}$ approach to networked control," IEEE Transactions on Automatic Control, vol. 50, no. 3, pp. 356-364, 2005.

[6] J. P. Hespanha, P. Naghshtabrizi, and Y. Xu, "A survey of recent results in networked control systems," Proceedings of the IEEE, vol. 95, no. 1, pp. 138-162, 2007.

[7] D. Huang and S. K. Nguang, "Robust disturbance attenuation for uncertain networked control systems with random time delays," IET Control Theory $\mathcal{E}$ Applications, vol. 2, no. 11, pp. 1008-1023, 2008.

[8] H. Gao and T. Chen, "Network-based $H_{\infty}$ output tracking control," IEEE Transactions on Automatic Control, vol. 53, no. 3, pp. 655-667, 2008.

[9] P. Wang, C.Z. Han, and B.C. Ding, "Stability of discrete-time networked control systems and its extension for robust $H_{\infty}$ control," International Journal of Systems Science. In press.

[10] K. C. Lee, S. Lee, and M. H. Lee, "Remote fuzzy logic control of networked control system via profibus-DP," IEEE Transactions on Industrial Electronics, vol. 50, no. 4, pp. 784-792, 2003.

[11] T. Li and Y. Fujimoto, "Control system with high-speed and real-time communication links," IEEE Transactions on Industrial Electronics, vol. 55, no. 4, pp. 1548-1557, 2008.

[12] S. Chai, G. P. Liu, D. Rees, and Y. Xia, "Design and practical implementation of internet-based predictive control of a servo system," IEEE Transactions on Control Systems Technology, vol. 16, no. 1, pp. 158-168, 2008.

[13] W. Leonhard, "Microcomputer control of high dynamic performance ac-drives-A survey," Automatica, vol. 22, no. 1, pp. 1-19, 1986.

[14] M. N. Uddin, M. A. Abido, and M. A. Rahman, "Development and implementation of a hybrid intelligent controller for interior permanent-magnet synchronous motor drives," IEEE Transactions on Industry Applications, vol. 40, no. 1, pp. 68-76, 2004.

[15] M. Yu, L. Wang, T. Chu, and F. Hao, "An LMI approach to networked control systems with data packet dropout and transmission delays," in Proceedings of the 43rd IEEE Conference on Decision and Control (CDC'04), pp. 3545-3550, December 2004. 
[16] Y. S. Lee, Y. S. Moon, W. H. Kwon, and P. G. Park, “Delay-dependent robust $H_{\infty}$ control for uncertain systems with a state-delay," Automatica, vol. 40, no. 1, pp. 65-72, 2004.

[17] Y. S. Moon, P. Park, W. H. Kwon, and Y. S. Lee, "Delay-dependent robust stabilization of uncertain state-delayed systems," International Journal of Control, vol. 74, no. 14, pp. 1447-1455, 2001. 


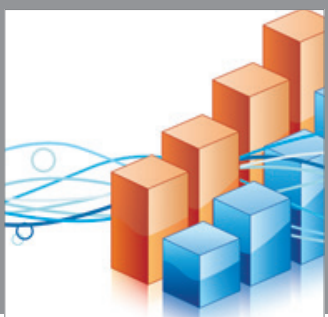

Advances in

Operations Research

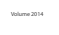

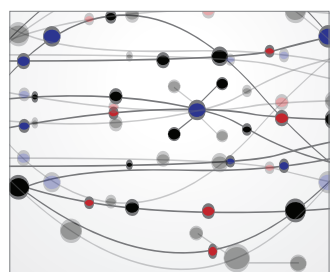

\section{The Scientific} World Journal
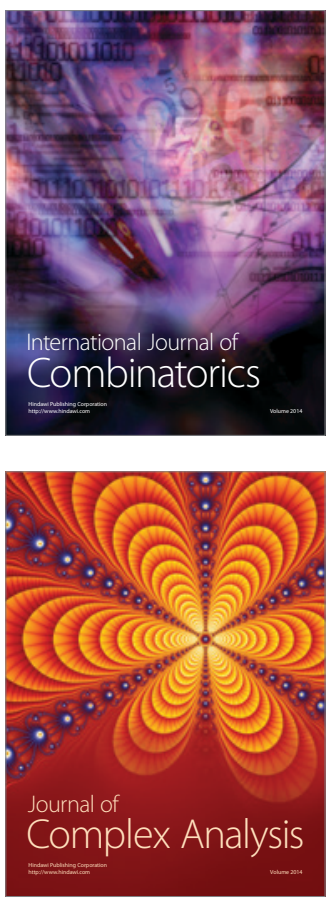

International Journal of

Mathematics and

Mathematical

Sciences
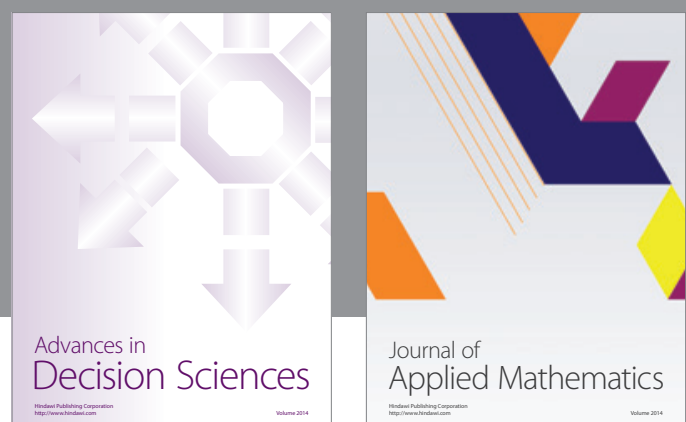

Journal of

Applied Mathematics
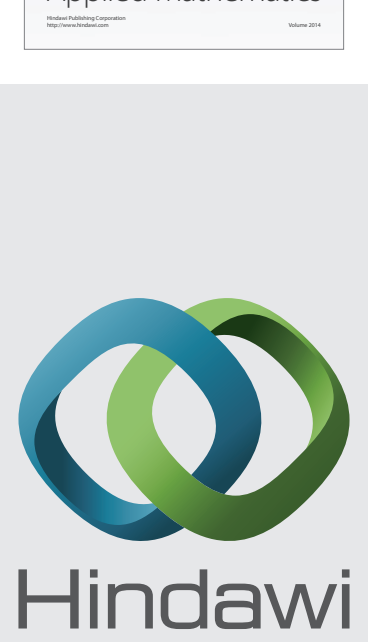

Submit your manuscripts at http://www.hindawi.com
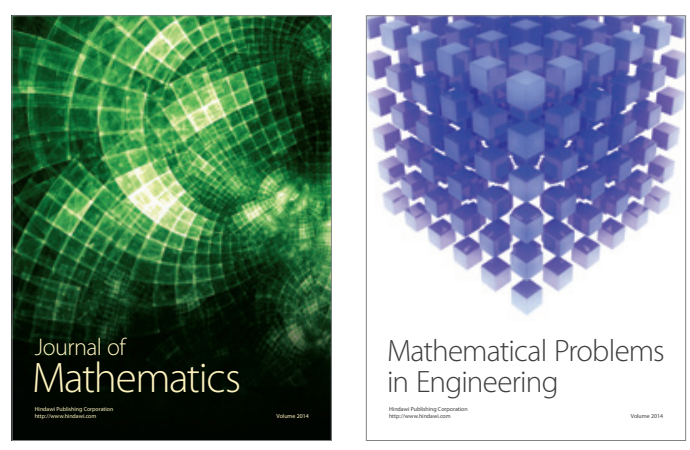

Mathematical Problems in Engineering
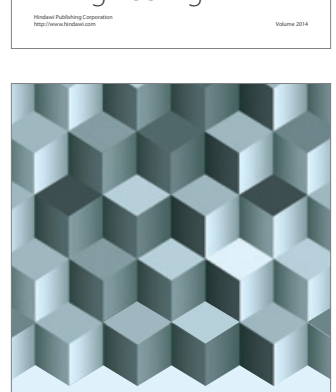

Journal of

Function Spaces
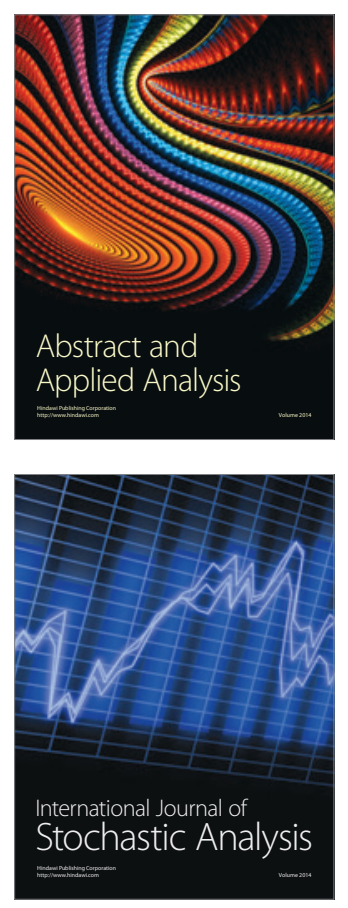

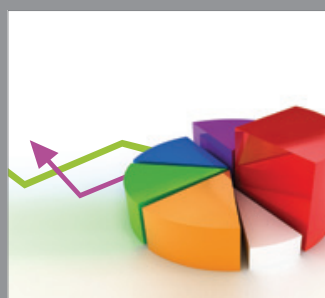

ournal of

Probability and Statistics

Promensencen
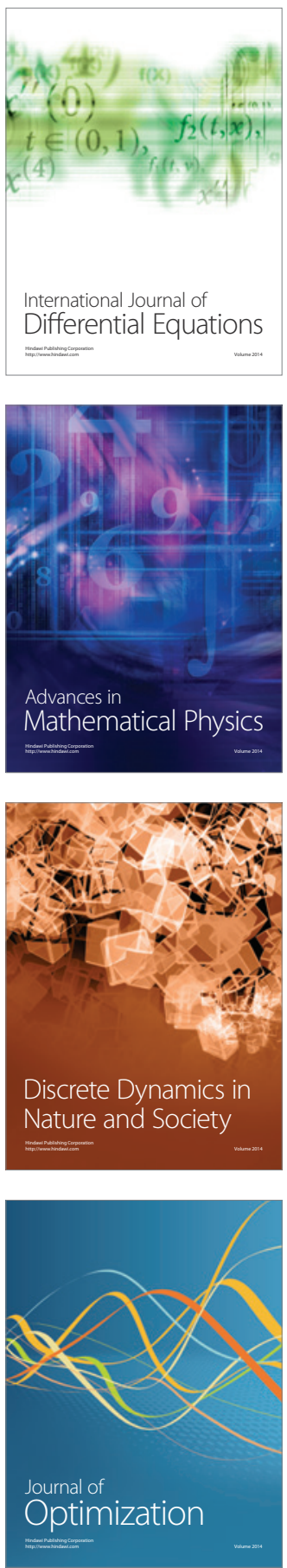